\title{
THE SUFFICIENT CONDITION FOR A CONVEX BODY TO ENCLOSE ANOTHER IN $\mathbb{R}^{4}$
}

\author{
JIAZU ZHOU
}

(Communicated by Christopher Croke)

\begin{abstract}
We follow Hadwiger and Ren's ideas to estimate the kinematic measure of a convex body $D_{1}$ with $C^{2}$-boundary $\partial D_{1}$ moving inside another convex body $D_{0}$ with the same kind of boundary $\partial D_{0}$ under the isometry group $G$ in $\mathbb{R}^{4}$. By using Chern and Yen's kinematic fundamental formula, C-S. Chen's kinematic formula for the total square mean curvature $\int_{\partial D_{0} \cap g \partial D_{1}} H^{2} d v$, and some well-known results about the curvatures of the 2-dimensional intersection submanifold $\partial D_{0} \cap g \partial D_{1}$, we obtain a sufficient condition to guarantee that one convex body can enclose another in $\mathbb{R}^{4}$.
\end{abstract}

\section{INTRODUCTION}

Many mathematicians have been interested in getting sufficient conditions to insure that a given domain $D_{1}$ of surface area $F_{1}$, bounded by boundary $\partial D_{1}$, of volume $V_{1}$ can be contained in a second domain $D_{0}$ of surface area $F_{0}$, bounded by boundary $\partial D_{0}$, of volume $V_{0}$ in an $n$-dimensional Euclidean space $\mathbb{R}^{n}$. The type of condition sought is meaningful if it is 'intrinsic' or 'extrinsic', i.e., if it only depends on the volumes of $D_{0}, D_{1}$, the areas of the boundaries $\partial D_{0}, \partial D_{1}$, and some curvature invariants of $D_{0}, D_{1}$. Hadwiger $[4,5]$ was first to use the method of integral geometry to obtain some sufficient conditions for one domain to contain another in the Euclidean plane $\mathbb{R}^{2}$. Ren [3] derived another condition in $\mathbb{R}^{2}$. It is natural to try to generalize Hadwiger's theorem to higher-dimensional Euclidean space $\mathbb{R}^{n}(n \geq 3)$. For a long time (before Zhang [9] and our works [10-14]) there was no general result for $\mathbb{R}^{n}(n \geq 3)$. Even restricted results, with some strong conditions placed on the two domains involved (for example, convexity and some topological conditions), were not available. Hadwiger and Ren used Blaschke's and Poincaré's formulas to estimate the kinematic measure of one domain moving into another under rigid motions in $\mathbb{R}^{2}$. However, there is no general Poincaré formula in $\mathbb{R}^{n}(n \geq 3)$ which can be used in our discussion. There is a general (or generalized) Blaschke's formula in $\mathbb{R}^{n}$ [1], i.e., Chern and Yen's kinematic fundamental formula. Our strategy is to find another formula to substitute for Poincaré's

Received by the editors January 30, 1992 and, in revised form, October 2, 1992.

1991 Mathematics Subject Classification. Primary 52A22, 53C05; Secondary 51M16, 60D05.

Key words and phrases. Kinematic density, kinematic formula, kinematic measure, convex body, domain, mean curvature, scalar curvature. 
formula, upon which Hadwiger and Ren's results in $\mathbb{R}^{2}$ are based. There are some different extensions of Poincaré's formula [1], but none of them are applicable for the situation of $\mathbb{R}^{n}$. In [11] the author used C-S. Chen's formula [6] to substitute for Poincaré's formula and obtained two different conditions in $\mathbb{R}^{3}$ by estimating the arc length of the intersection curve $\partial D_{0} \cap g \partial D_{1}$ of two boundaries $\partial D_{0}$ and $\partial D_{1}$ of the two convex bodies involved. In [10] we obtained a sufficient condition for $n$-dimensional $(n \geq 4)$ Euclidean space $\mathbb{R}^{n}$ by using the kinematic formula for the total scalar curvature $\int_{\partial D_{0} \cap g \partial D_{1}} R d v$ of the $(n-2)$-dimensional submanifold $\partial D_{0} \cap g \partial D_{1}$ to substitute for Poincaré's formula. Later, we removed the convexity restriction on the domains and got some analogues of Hadwiger's theorem in space $\mathbb{R}^{3}$ (see [12-14]).

In this paper we restrict our discussion to $\mathbb{R}^{4}$. There the situation is very concrete, and there are some well-known results we can use for the 2-dimensional intersection manifold $\partial D_{0} \cap g \partial D_{1}$ of two boundaries $\partial D_{0}$ and $\partial D_{1}$. We use Chern and Yen's kinematic fundamental formula and Chen's general formulas $[1,6]$ to substitute for Blaschke and Poincaré's formulas and obtain a sufficient condition for $\mathbb{R}^{4}$. Our work is a direct application of the higherdimensional Chern and Yen's formula. For a discussion of the situation in $\mathbb{R}^{3}$ and $\mathbb{R}^{n}(n \geq 4)$, see [10-14].

\section{Preliminaries}

Let $M^{p}, M^{q}$ be a pair of closed submanifolds in Euclidean space $\mathbb{R}^{n}$. We assume $p+q \geq n+1$ so that generically $M^{p} \cap g M^{q}$ is a submanifold of dimension $p+q-n$ for every $g \in G$, the group of motions in $\mathbb{R}^{n}$. Let $M$ be a submanifold of $\mathbb{R}^{n}$. The second fundamental form of $M$ at $x \in M$ is a symmetric bilinear mapping

$$
T: M_{x} \times M_{x} \longrightarrow M_{x}^{\perp},
$$

where $M_{x}$ is the tangent space of $M$ at $x$ and $M_{x}^{\perp}$ is the normal space of $M$ at $x$. Let $\nabla$ be the covariant derivative of $\mathbb{R}^{n}$ so that the directional derivative of a vector field $X$ along another $Y$ is $\nabla_{Y} X$. If $M$ is immersed in $\mathbb{R}^{n}$ and $X, Y$ are tangent to $M$, then at $x \in M$

$$
T_{X} Y=\text { normal component of } \nabla_{X} Y \text { with respect to } M_{x} \text {. }
$$

It can be verified that $T$ is a symmetric bilinear tensor. We shall denote the second fundamental form of $M^{p}$ by $T^{p}$, where the superscript $p$ is the dimension of the submanifold. For a smooth curve $C$ in $\mathbb{R}^{n}, T^{1}$ has the following interpretation. Let $t$ be a unit vector tangent to $C ; \kappa=\left\|T_{t}^{1} t\right\|$ will be the curvature of $C$, and $n=T_{t}^{1} t / \kappa$ will be the normal of $C$. These definitions agree with the classical theory of curves in $\mathbb{R}^{3}$.

From now on, we assume that $G$ is the isometry group in $\mathbb{R}^{n}$ and $d g$ the kinematic density for $\mathbb{R}^{n}$. Let $H(\cdot)$ and $R(\cdot)$, respectively, be the mean curvature and scalar curvature of the submanifold. Assume $\tau\left(M^{m}\right)$ is defined by

$$
\text { (3) } \tau\left(M^{m}\right)=\frac{3 m}{m+2} O_{m-1} \int_{M^{m}} H^{2}\left(M^{m}\right) d v-\frac{4}{m(m+2)} O_{m-1} \int_{M^{m}} R\left(M^{m}\right) d v \text {, }
$$


where $O_{m}$ is the surface area of the $m$-dimensional unit sphere and its value is given by

$$
O_{m}=\frac{2 \pi^{(m+1) / 2}}{\Gamma((m+1) / 2)}
$$

Then C-S. Chen's theorem [6] says

$$
\begin{gathered}
\int_{\left\{g: M^{p} \cap g M^{q} \neq \varnothing\right\}} \tau\left(M^{p} \cap g M^{q}\right) d g \\
=C_{0} \tau\left(M^{p}\right) F\left(M^{q}\right)+C_{2} \tau\left(M^{q}\right) F\left(M^{p}\right),
\end{gathered}
$$

where $F(\cdot)$ is the volume function and

$$
\begin{aligned}
& C_{0}=\frac{O_{n} \cdots O_{0} O_{q-1} O_{p+q-n+1} O_{p+q-n}}{O_{0} O_{p-1} O_{p} O_{q+1} O_{q}} \\
& C_{2}=\frac{O_{n} \cdots O_{0} O_{p-1} O_{p+q-n+1} O_{p+q-n}}{O_{0} O_{q-1} O_{q} O_{p+1} O_{p}}
\end{aligned}
$$

In [2] the kinematic formula for

$$
\mu_{2}\left(M^{m}\right)=\frac{1}{m(m-1)} \tilde{R}\left(M^{m}\right)=\frac{1}{m(m-1)} \int_{M^{m}} R d v
$$

where $m=\operatorname{dim} M$, reads

$$
\begin{aligned}
\frac{1}{(p+q-n)(p+q-n-1)} \int_{\left\{g: M^{p} \cap g M^{q} \neq \varnothing\right\}} \tilde{R}\left(M^{p} \cap g M^{q}\right) d g \\
=\frac{C_{0}}{p(p-1)} \frac{O_{p-1}}{O_{p+q-n-1}} \tilde{R}\left(M^{p}\right) F\left(M^{q}\right) \\
\quad+\frac{C_{2}}{q(q-1)} \frac{O_{q-1}}{O_{p+q-n-1}} \tilde{R}\left(M^{q}\right) F\left(M^{p}\right) .
\end{aligned}
$$

Let $\tilde{H}\left(M^{m}\right)$ and $\tilde{R}\left(M^{m}\right)$ denote the total square of mean curvature and total scalar curvature of $M^{m}$, respectively, i.e.,

$$
\tilde{H}\left(M^{m}\right)=\int_{M^{m}} H^{2}\left(M^{m}\right) d v, \quad \tilde{R}\left(M^{m}\right)=\int_{M^{m}} R\left(M^{m}\right) d v .
$$

After a little calculation, we obtain the kinematic formula

$$
\begin{aligned}
\int_{\left\{g: M^{p} \cap g M^{q} \neq \varnothing\right\}} \tilde{H}\left(M^{p} \cap g M^{q}\right) d g \\
=\frac{C_{0}}{(p+2)(p+q-n)} \frac{O_{p-1}}{O_{p+q-n-1}} \\
\quad \times\left[p(p+q-n+2) \tilde{H}\left(M^{p}\right)-\frac{4(n-q)}{p(p-1)} \tilde{R}\left(M^{p}\right)\right] F\left(M^{q}\right) \\
\quad+\frac{C_{2}}{(q+2)(p+q-n)} \frac{O_{q-1}}{O_{p+q-n-1}} \\
\quad \times\left[q(p+q-n+2) \tilde{H}\left(M^{q}\right)-\frac{4(n-p)}{q(q-1)} \tilde{R}\left(M^{q}\right)\right] F\left(M^{p}\right) .
\end{aligned}
$$


Let the boundary $\partial D$ of a convex body $D$ be a hypersurface of class $C^{2}$. It is known that at each point of a hypersurface $\Sigma$ in $\mathbb{R}^{n}$ there are $n-1$ principal directions and $n-1$ principal curvatures $\kappa_{1}, \kappa_{2}, \ldots, \kappa_{n-1}$. If $d v$ denotes the area element of $\Sigma$, then the $r$ th integral of mean clirvature is defined by

$$
M_{r}(\Sigma)=\left(\begin{array}{c}
n-1 \\
r
\end{array}\right)^{-1} \int_{\Sigma}\left\{\kappa_{i_{1}}, \kappa_{i_{2}}, \ldots, \kappa_{i_{r}}\right\} d v,
$$

where $\left\{\kappa_{i_{1}}, \kappa_{i_{2}}, \ldots, \kappa_{i_{r}}\right\}$ denotes the $r$ th elementary symmetric function of the principal curvatures. In particular, $M_{0}$ is the area, and $M_{n-1}$ is a numerical multiple of the degree of mapping of $\Sigma$ into the unit hypersphere defined by the field of normals. Let $D_{0}$ and $D_{1}$ be two convex bodies in $\mathbb{R}^{n}$ bounded by the hypersurfaces $\partial D_{0}$ and $\partial D_{1}$, which we assume to be of class $C^{2} \cdot\left(M^{0}\right)_{i}$, $\left(M^{1}\right)_{i}$ are the $i$ th integrals of mean curvature of $\partial D_{0}$ and $\partial D_{1}$, respectively. For simplicity we will denote these by $M_{i}^{0}$ and $M_{i}^{1}$. Chern and Yen's kinematic fundamental formula [1] is

$$
\begin{aligned}
\left.\int_{\{g:} D_{0} \cap g D_{1} \neq \varnothing\right\} & d g \\
& =O_{n-2} \cdots O_{1}\left[O_{n-1}\left(V_{0}+V_{1}\right)+\frac{1}{n} \sum_{h=0}^{n-2}\left(\begin{array}{c}
n \\
h+1
\end{array}\right) M_{h}^{0} M_{n-2-h}^{1}\right] .
\end{aligned}
$$

Let $M$ be an $n$-dimensional closed submanifold in Euclidean space $\mathbb{R}^{m}, H$ the mean curvature of $M$. Then we have B-Y. Chen's formula [7]

$$
\int_{M}|H|^{n} d v \geq O_{n}
$$

Equality holds in (11) precisely when $M$ is embedded as an $n$-sphere of $\mathbb{R}^{m}$.

\section{MAIN THEOREM}

Theorem 1. Let $D_{i}(i=0,1)$ be convex bodies in 4-dimensional Euclidean space $\mathbb{R}^{4}$, with $C^{2}$ boundaries $\partial D_{i}$, and let $V_{i}, F_{i}, M_{r}^{i}, \tilde{H}_{i}, \tilde{R}_{i}$ be the volume of $D_{i}$, the surface area of $D_{i}$, the rth integral of mean curvature of $\partial D_{i}$, the total square mean curvature, and integral of scalar curvature of $\partial D_{i}$, respectively. $H_{i}$ and $R_{i}$ represent, respectively, the mean curvature and scalar curvature of $\partial D_{i}$. Then a sufficient condition for $D_{0}$ to contain $D_{1}$ or for $D_{1}$ to contain $D_{0}$ is

$$
\begin{aligned}
& {\left[2 \pi^{2}\left(V_{0}+V_{1}\right)+F_{0} M_{2}^{1}+F_{1} M_{2}^{0}+\frac{3}{2} M_{1}^{0} M_{1}^{1}\right]} \\
& \quad-\frac{2}{15}\left[\left(18 \tilde{H}_{0}-\tilde{R}_{0}\right) F_{1}+\left(18 \tilde{H}_{1}-\tilde{R}_{1}\right) F_{0}\right]>0 .
\end{aligned}
$$

Moreover,

(1) if $V_{1} \geq V_{0}$, then $D_{1}$ can contain $D_{0}$;

(2) if $V_{1} \leq V_{0}$, then $D_{1}$ can be contained in $D_{0}$.

This formula comes from estimating the kinematic measure of the set of rigid 
motions which move one convex body inside another in $\mathbb{R}^{4}$, i.e.,

$$
\begin{aligned}
m\left\{g \in G: g D_{1} \subseteq D_{0} \text { or } g D_{0} \subseteq D_{1}\right\} & d g-\int_{\left\{g: \partial D_{0} \cap g \partial D_{1} \neq \varnothing\right\}} d g \\
= & \int_{\left\{g: D_{0} \cap g D_{1} \neq \varnothing\right\}} d g \\
\geq & 8 \pi^{2}\left[2 \pi^{2}\left(V_{0}+V_{1}\right)+F_{0} M_{2}^{1}+F_{1} M_{2}^{0}+\frac{3}{2} M_{1}^{0} M_{1}^{1}\right] \\
& -\frac{16 \pi^{2}}{15}\left[\left(18 \tilde{H}_{0}-\tilde{R}_{0}\right) F_{1}+\left(18 \tilde{H}_{1}-\tilde{R}_{1}\right) F_{0}\right] .
\end{aligned}
$$

The equality holds if and only if the two convex bodies are balls.

\section{THE PROOF OF THE MAIN RESULT}

First, we estimate the integral

$$
\Phi=\int_{\left\{g: \partial D_{0} \cap g \partial D_{1} \neq \varnothing\right\}} d g .
$$

For each $g \in G$, the intersection $\partial D_{0} \cap g \partial D_{1}$ may be composed of several connected components, i.e., $\partial D_{0} \cap g \partial D_{1}=\sum_{i=1}^{N_{g}} M_{i}$, where each $M_{i}$ is a connected closed surface and $N_{g}$ is always finite and only depends on $g$. By applying B-Y. Chen's formula (11) to our generic 2-dimensional submanifold $\partial D_{0} \cap g \partial D_{1}$ we have

$$
\int_{\partial D_{0} \cap g \partial D_{1}} H^{2} d v \geq 4 \pi
$$

For fixed $g$, equality holds in (15) if and only if $\partial D_{0} \cap g \partial D_{1}$ is a 2-sphere in $\mathbb{R}^{4}$ (B-Y. Chen [7]). If equality holds in (15) for every $g$ then the two convex bodies $D_{0}$ and $D_{1}$ must be balls (a result of P. Goodey $\left.[15,16]\right)$. By $(15)$ and (8) we have

$$
\begin{aligned}
4 \pi \int_{\left\{g: \partial D_{0} \cap g \partial D_{1} \neq \varnothing\right\}} d g & \leq \int_{\left\{g: \partial D_{0} \cap g \partial D_{1} \neq \varnothing\right\}}\left(\int_{\partial D_{0} \cap g \partial D_{1}} H^{2} d v\right) d g \\
& =\frac{64 \pi^{3}}{15}\left[\left(18 \tilde{H}_{0}-\tilde{R}_{0}\right) F_{1}+\left(18 \tilde{H}_{1}-\tilde{R}_{1}\right) F_{0}\right]
\end{aligned}
$$

i.e.,

$$
\Phi=\int_{\left\{g: \partial D_{0} \cap g \partial D_{1} \neq \varnothing\right\}} d g \leq \frac{16 \pi^{2}}{15}\left[\left(18 \tilde{H}_{0}-\tilde{R}_{0}\right) F_{1}+\left(18 \tilde{H}_{1}-\tilde{R}_{1}\right) F_{0}\right] .
$$

Using Chern and Yen's formula (10) and (17) we obtain

$$
\begin{aligned}
& m\left\{g \in G: g D_{1} \subseteq D_{0} \text { or } g D_{0} \subseteq D_{1}\right\} \\
&= \int_{\left\{g: D_{0} \cap g D_{1} \neq \varnothing\right\}} d g-\int_{\left\{g: \partial D_{0} \cap g \partial D_{1} \neq \varnothing\right\}} d g \\
& \geq 8 \pi^{2}\left[2 \pi^{2}\left(V_{0}+V_{1}\right)+F_{0} M_{2}^{1}+F_{1} M_{2}^{0}+\frac{3}{2} M_{1}^{0} M_{1}^{1}\right] \\
&-\frac{16 \pi^{2}}{15}\left[\left(18 \tilde{H}_{0}-\tilde{R}_{0}\right) F_{1}+\left(18 \tilde{H}_{1}-\tilde{R}_{1}\right) F_{0}\right]
\end{aligned}
$$


As before, the case of equality in (16), i.e., (17) and (18), hold if the 2dimensional manifold $\partial D_{0} \cap g \partial D_{1}$ is a 2-sphere in $\mathbb{R}^{4}$ (in particular, is connected), and if this holds for almost every $g$ then the convex bodies $D_{0}$ and $D_{1}$ are balls. Equality in (18) has the same consequences.

\section{REMARKS}

1. Let $D_{0}$ and $D_{1}$ be two domains in $\mathbb{R}^{4}$ bounded by the hypersurfaces $\partial D_{0}$ and $\partial D_{1}$, which we assume to be of class $C^{2}$. Denote by $\chi(\cdot)$ the EulerPoincaré characteristic. We have Chern and Yen's kinematic formula [1]

$$
\begin{aligned}
\int_{\left\{g: D_{0} \cap g D_{1} \neq \varnothing\right\}} \chi\left(D_{0} \cap g D_{1}\right) d g & \\
=O_{n-2} \cdots O_{1}\left[O_{n-1}\left(\chi\left(D_{0}\right) V_{1}+\chi\left(D_{1}\right) V_{0}\right)\right. & \\
& \left.+\frac{1}{n} \sum_{h=0}^{n-2}\left(\begin{array}{c}
n \\
h+1
\end{array}\right) M_{h}^{0} M_{n-h-2}^{1}\right] .
\end{aligned}
$$

Then we have

Theorem 2. Let $D_{i}(i=0,1)$ be two domains in 4-dimensional Euclidean space $\mathbb{R}^{4}$ bounded by the $C^{2}$-smooth boundaries $\partial D_{i}$. Suppose that $V_{i}, F_{i} M_{r}^{i}$, $H_{i}, \quad R_{i}, \tilde{H}_{i}, \tilde{R}_{i}$ are as in Theorem 1 . Denote by $\chi(\cdot)$ the Euler-Poincaré characteristic. Moreover, assume that for all rigid motion $g \in G$ in $\mathbb{R}^{4}$, $\chi\left(D_{0} \cap g D_{1}\right) \leq n_{0}$, a finite integer. Then a sufficient condition for $D_{1}$ to enclose, or to be enclosed in, $D_{0}$ is

$$
\begin{gathered}
2 \pi^{2}\left(\chi\left(D_{0}\right) V_{1}+\chi\left(D_{1}\right) V_{0}\right)+F_{0} M_{2}^{1}+F_{1} M_{2}^{0}+\frac{3}{2} M_{1}^{0} M_{1}^{1} \\
-\frac{2 n_{0}}{15}\left[\left(18 \tilde{H}_{0}-\tilde{R}_{0}\right) F_{1}+\left(18 \tilde{H}_{1}-\tilde{R}_{1}\right) F_{0}\right]>0 .
\end{gathered}
$$

Moreover,

(1) if $V_{1} \geq V_{0}$, then $D_{1}$ can enclose $D_{0}$;

(2) if $V_{1} \leq V_{0}$, then $D_{1}$ can be enclosed in $D_{0}$.

(20) comes from estimating the kinematic measure of one domain moving into another under the rigid motions in $\mathbb{R}^{4}$, i.e.,

$$
\begin{aligned}
& m\left\{g \in G: g D_{1} \subset D_{0} \text { or } g D_{0} \subset D_{1}\right\} \\
&=\int_{\left\{g: D_{0} \cap g D_{1} \neq \varnothing\right\}} d g-\int_{\left\{g: \partial D_{0} \cap g \partial D_{1} \neq \varnothing\right\}} d g \\
& \geq \\
& \geq \frac{8 \pi^{2}}{n_{0}}\left[2 \pi^{2}\left(\chi\left(D_{0}\right) V_{1}+\chi\left(D_{1}\right) V_{0}\right)+F_{0} M_{2}^{1}+F_{1} M_{2}^{0}+\frac{3}{2} M_{1}^{0} M_{1}^{1}\right] \\
&-\frac{16 \pi^{2}}{15}\left[\left(18 \tilde{H}_{0}-\tilde{R}_{0}\right) F_{1}+\left(18 \tilde{H}_{1}-\tilde{R}_{1}\right) F_{0}\right] .
\end{aligned}
$$

If $D_{0}$ and $D_{1}$ are convex bodies, we have $\chi\left(D_{0}\right)=\chi\left(D_{1}\right)=\chi\left(D_{0} \cap g D_{1}\right)=$ $n_{0}=1$ and Theorem 2 becomes Theorem 1 .

2. It would be interesting to remove the "smooth" restriction from the convex bodies involved in Theorem 1 . All the notions except $\tilde{H}$ here are well defined 
for nonsmooth convex bodies. In fact, we can use Quermassintegrals to substitute for $M_{r}^{i}$. If we could find other substitutions for $\tilde{H}$, the results in this paper can be interpreted for arbitrary convex bodies. This is definitely worth thinking about.

3. Of course, the sufficient conditions (12) and (20) are not necessary.

\section{ACKNOWLEDGMENT}

The author thanks Professor Eric Grinberg for his teaching, support, and encouragement. He also thanks Professors R. Howard, C. C. Hsiung, Ren Delin, and Yang Wenmao for their support and encouragement. He also expresses his sincere thanks to Professor E. Lutwak for helpful discussions during his visit to Temple in November, 1991. The author is indebted to Professor P. Goodey's comments and suggestions. Mr. Zhang Gaoyong's comments are appreciated. Finally, the author thanks the referee for useful suggestions.

\section{REFERENCES}

1. L. A. Santaló, Integral geometry and geometric probability, Addison-Wesley, Reading, MA, 1976.

2. S. S. Chern, On the kinematic formula in the euclidean space of $n$ dimensions, Amer. J. Math. 74 (1952), 227-236.

3. Delin Ren, Introduction to Integral Geometry, Shanghai Press of Sciences and Technology, 1987.

4. H. Hadwiger, Überdeckung ebener Bereiche durch Kreise und Quadrate, Comment. Math. Helv. 13 (1941), 195-200.

5. H. Hadwiger, Gegenseitige Bedeckbarkeit zweier Eibereiche und Isoperimetrie, Viertejschr. Naturforsch. Gesellsch. Zürich 86 (1941), 152-156.

6. C-S. Chen, On the kinematic formular of square of mean curvature, Indiana Univ. Math. J. 22 (1972-3), 1163-1169.

7. B-Y. Chen, Geometry of submanifold, Marcel Dekker, New York, 1973.

8. S. S. Chern, A simple intrinsic proof of the Gauss-Bonnet formula for closed Riemannian manifolds, Ann. of Math. (2) 45 (1944), 747-752.

9. Gaoyong Zhang, $A$ sufficient condition for one convex body containing another, Chinese Ann. Math. Ser. B 9 (1988), 447-451.

10. Jiazu Zhou, Analogues of Hadwiger's theorem in space $\mathbb{R}^{n}$ and sufficient conditions for a convex domain to enclose another, submitted.

11. __ Generalizations of Hadwiger's theorem and sufficient conditions for a convex domain to fit another in $\mathbb{R}^{3}$, submitted.

12. __ A kinematic formula and analogues of Hadwiger's theorem in space, Contemp. Math., vol. 140, Amer. Math. Soc., Providence, RI, 1992, pp. 159-167.

13. _ When can one domain enclose another in space, J. Austral. Math. Soc. Ser. A (to appear).

14. _ Kinematic formulas for the power of mean curvature and Hadwiger's theorem in space, Trans. Amer. Math. Soc. (to appear).

15. P. Goodey, Connectivity and free rolling convex bodies, Mathematika 29 (1982), 249-259.

16. __ Homothetic ellipsoids, Math. Proc. Cambridge Philos. Soc. 93 (1983), 25-34.

Department of Mathematics, Temple University, Philadelphia, Pennsylvania 19122

E-mail address: zhou@euclid.math.temple.edu 